\title{
SURVEY OF INTELLIGENT CONTROL ALGORITHMS FOR HUMANOID ROBOTS
}

\author{
Dusko Katić Miomir Vukobratović
}

\author{
Robotics Laboratory, Mihailo Pupin Institute \\ Volgina 15, 11060 Belgrade, Serbia $\& 3$ Montenegro \\ telephone: (+381)-11-2776-222; fax: $(+381)-11-775-870$ \\ e-mail:dusko@robot.imp.bg.ac.yu,vuk@robot.imp.bg.ac.yu
}

\begin{abstract}
This paper focusses on the application of intelligent control techniques (neural networks, fuzzy logic and genetic algorithms) and their hybrid forms (neuro-fuzzy networks, neuro-genetic and fuzzy-genetic algorithms) in the area of humanoid robotic systems. Overall, this survey covers a broad selection of examples that will serve to demonstrate the advantages and disadvantages of the application of intelligent control techniques.
\end{abstract}

Keywords: Humanoid Robots, Neural Networks, Fuzzy Logic, Genetic Algorithms

\section{INTRODUCTION}

Many aspects of modern life involve the use of intelligent machines capable of operating under dynamic interaction with their environment. In view of this, the field of biped locomotion is of special interest when human-like robots are concerned. Humanoid robots as anthropomorphic walking machines have been in operation for more than twenty years. Currently, research on humanoid robots and biped locomotion is one of the most exciting topics in the field of robotics. There are more than 50 major humanoid robot projects around the world, along with many other bipedal walking projects (an extensive list of projects is given at the site www.androidworld.com). Humanoid robot applications usually demand the robot be highly intelligent. Intelligent humanoid robots are functionally oriented devices, built to perform sets of tasks instead of humans. They are autonomous systems capable of extracting information from their environments and using knowledge about the world and intelligence of their duties and proper governing capabilities. Intelligent humanoid robots should be autonomous to move safely in a meaningful and purposive manner, i.e. to accept high-level descriptions of tasks (specifying what the user wants to be done, rather than how to do it) and would execute them without further human intervention.

Naturally, the first approach to making humanoid robots more intelligent was the integration of sophisticated sensor systems as computer vision, tactile sensing, ultrasonic and sonar sensors, laser scanners and other smart sensors. However, today's sensor products are still very limited in interactivity and adaptability to changing environments. A major reason is that uncertainty and dynamic changes make the development of reliable artificial systems particularly challenging. On the other hand, to design robots and systems that best adapt to their environment, the necessary research includes investigations in the field of mechanical robot design (intelligent mechanics), environment perception systems and embedded intelligent control that ought to cope with the task complexity, multi-objective decision making, large volume of perception data and substantial amount of heuristic information. Also, in the case when the robot performs in an unknown environment, the knowledge may not be sufficient. Hence, the 
robot has to adapt to the environment and to be capable of acquiring new knowledge through the process of learning.

There are several intelligent paradigms that are capable of solving intelligent control problems in humanoid robotics. Connectionist theory (NN neural networks), fuzzy logic (FL), and theory of evolutionary computation (GA - genetic algorithms), are of great importance in the development of intelligent humanoid robot control algorithms. Due to their strong learning and cognitive abilities and good tolerance of uncertainty and imprecision, intelligent techniques have found wide applications in the area of advanced control of humanoid robots. Also, of great importance in the development of efficient algorithms are the hybrid techniques based on the integration of particular techniques such as neuro-fuzzy networks, neurogenetic algorithms and fuzzy-genetic algorithms. Each of the proposed paradigms has its own merits and drawbacks. To overcome the drawbacks, certain integration and synthesis of hybrid techniques (symbiotic intelligence) are needed for efficient application in humanoid robotics.

\section{CONTROL PROBLEMS IN HUMANOID ROBOTICS}

In spite of a significant progress and accomplishments achieved in the design of a hardware platform of humanoid robot and synthesis of advanced intelligent control of humanoid robots, a lot of work has still to be done in order to improve actuators, sensors, materials, energy accumulators, hardware, and control software that can be utilized to realize user-friendly humanoid robots.

Previous studies of biological nature, theoretical and computer simulation, have focussed on the structure and selection of control algorithms according to different criteria such as energy efficiency, energy distribution along the time cycle, stability, velocity, comfort, mobility, and environment impact. Nevertheless, in addition to these aspects, it is also necessary to consider some other issues: capability of mechanical implementation due to the physical limitations of joint actuators, coping with complex highly-nonlinear dynamics and uncertainties in the model-based approach, complex nature of periodic and rhythmic gait, inclusion of learning and adaptation capabilities, computation issues, etc.

Irrespective of the humanoid robot structure and complexity, the basic characteristic of all bipedal systems are: a) the DOF formed between the foot and the ground is unilateral and underactuated (Goswami (1999)) ; b) the gait repeatability (symmetry) and regular interchangeability of the number of legs that are simultaneously in contact with the ground. During the walk, two different situations arise in sequence: the statically stable double-support phase in which the mechanism is supported on both feet simultaneously, and statically unstable single-support phase when only one foot of the mechanism is in contact with the ground. Also, it is well known that through the process of running the robot can be most of the time in no-support phase. In this case, the control schemes that are successful for walking problem are not necessarily successful for the running problem.

The stability issues of humanoid robot walking are the crucial point in the process of control synthesis. In view of this humanoid walking robots can be classified in three different categories (Marchese et al. (2001)). First category represents static walkers, whose motion is very slow so that the system's stability is completely described by the normal projection of the Center of Gravity, which only depends on the joint's position. Second category represents dynamic walkers, biped robots with feet and actuated ankles. Postural stability of dynamic walkers depends on joint's velocities and acceleration too. The third category represents purely dynamic walkers, robots without feet. In this case the support polygon during the singlesupport phase is reduced to a point, so that static walking is not possible. For all the mentioned categories of walking robots, the issue of stable and reliable bipedal walk is the most fundamental and yet unsolved with a high degree of reliability. This subject has been studied mainly through the following two classes of walking pattern generators and robot controllers. The first approach is to generate a dynamically consistent periodic walking pattern off-line. It is done assuming that the models of robot and environment are available, and the kinematic and dynamic parameters of the robot model are precisely defined. On the other hand, the second approach uses limited or simplified knowledge of the system's dynamics (Raibert (1986); Zheng and Shen (1990)). However, in this case, the control relies much on the feedback control, and it is necessary to develop methods without high computation resources for real-time implementation.

The rotational equilibrium of the foot is the major factor of postural instability with legged robots. The question has motivated the definition of several dynamic-based criteria for the evaluation and control of balance in biped locomotion. The most common criteria are the centre of pressure (CoP), the zero-moment point (ZMP) and the foot-rotation indicator (FRI), (Vukobratovic et al. (2002), Goswami (1999)). Of these criteria, the ZMP concept has gained widest acceptance and played a crucial role in solving the biped robot 
stability and periodic walking pattern synthesis (Vukobratovic et al. (2002)). The ZMP is defined as the point on the ground about which the sum of all the moments of the active forces equals zero. If the ZMP is within the convex hull of all contact points between the foot and the ground, the biped robot can walk.

For a legged robot walking on complex terrain, such as a ground consisting of soft and hard uneven parts, a statically stable walking manner is recommended. However, in the cases of soft terrain, up and down slopes or unknown environment, the walking machine may lose its stability because of the position planning errors and unbalanced foot forces. Hence, position control alone is not sufficient for practical walking, position/force control being thus necessary. Foot force control (Zhou and Low (2001)) can overcome these problems, so that foot force control is one of the ways to improve the terrain adaptability of walking robots.

A practical biped needs to be more like a human - capable of switching between different known gaits on familiar terrain and learning new gaits when presented with unknown terrain. In this sense, it seems essential to combine force control techniques with more advanced algorithms such as adaptive and learning strategies. When the ground conditions and stability constraint are satisfied, it is desirable to select a walking pattern that requires small torque and velocity of the joint actuators. Humanoid robots are inevitably restricted to a limited amount of energy supply. It would therefore be advantageous to consider the minimum energy consumption, when cyclic movements like walking are involved.

In summary, conventional control algorithms for humanoid robots can run into some problems related to mathematical tractability, optimisation, limited extendability and limited biological plausibility. The presented intelligent control techniques have a potential to overcome the mentioned constraints.

\section{CONNECTIONIST CONTROL ALGORITHMS IN HUMANOID ROBOTICS}

Recently, some researchers have begun considering the use of neural networks for control of humanoid walking (Doerschuk et al. (1998); Miller (1994); Miller (1987); Kun et al. (1999); Wang and Gruver (1992)). This approach makes possible the learning of new gaits which are not weighted combinations of predefined biped gaits. Various types of neural networks are used for gait synthesis and control design of humanoid robots such as multilayer perceptrons, CMAC (Cerebellar Model
Arithmetic Controller) networks, recurrent neural network, RBF (Radial Basis Function) networks or Hopfield networks, which are trained by supervised or unsupervised (reinforced) learning methods. The majority of the proposed control algorithms have been verified by simulation, while there were few experimental verifications on real biped and humanoid robots. Neural networks have been used as efficient tools for the synthesis and off-line and on-line adaptation of biped gait. Another important role of connectionist systems in control of humanoid robots has been the solving of static and dynamic balance during the process of walking and running on terrain with different environment characteristics.

Kitamura 1988 proposed a walking controller based on Hopfield neural network in combination with an inverted pendulum dynamic model. The optimization function of the Hopfield network was based on complete dynamic model of biped. Salatian et al. 1992a,1992b,1997 studied off-line and on-line reinforcement techniques for adapting the gait designed for horizontal surfaces to be executed on sloping surfaces. They considered humanoid robot SD-2 with 8 DOFs and two force sensors on both feet. These control algorithms without considering kinematic and dynamic models of humanoid robot were evaluated using a biped dynamic simulation. The control structure includes gait trajectory synthesizer) and neural networks that are tuned by reinforcement signal from force sensors at the feet. The joint positions of the robot are adjusted until the force sensors indicate that the robot has a stable gait. The neural network has the task to map the relation between foot forces and adjustment of the joint positions. The reinforcement learning is used because the neural network receives no direct instruction on which joint position needs to be modified. The neural network is not a conventional type of network (perceptrons) and includes a net of more neurons with inhibitory/excitory inputs from the sensor unit. Every joint of the robot is associated with a neuron called joint neuron. Using previously mentioned "regard-andpunish" strategy, the neural network converges quickly and generates a stable gait for the sloping surface. In this way, reinforcement learning is very attractive because the algorithm does not require an explicit feedback signal. Static and pseudodynamic learning are demonstrated to prove that the proposed mechanism is valid for robot walking on the sloping surface. In this approach, kinematic and dynamic models were not used, hence it would be a problem for real dynamic walking with a high speed. Also, the real terrain is more complex than the environments used in test experiments, so that more studies need to be conducted to make the robot walk robustly on different sorts of terrain. 
More recently, Miller et al. 1994,1987,1999 has developed a hierarchical controller that combines simple gait oscillators, classical feedback control techniques and neural network learning, and does not require detailed equations of the dynamics of walking. The emphasis is on the real-time control studies using an experimental ten-axis biped robot with foot force sensors. The neural network learning is achieved using CMAC controller, where CMAC neural networks were used essentially as context sensitive integral errors in the controller, the control context being defined by the CMAC input vector. There are 3 different CMAC neural networks for humanoid posture control. The Front/Back Balance CMAC neural network was used to provide front/back balance during standing, swaying and walking. The training of this network is realized using data from foot sensors. The second CMAC neural network is used for Right/Left Balance, to predict the correct knee extension required to achieve sufficient lateral momentum for lifting the corresponding foot for the desired length of time. The training of this network is realized using temporal difference method based on the difference between the desired and real time of foot rising. The third CMAC network is used to learn kinematically consistent robot postures. In this case, training is also realized by data from foot sensors.

The results indicated that the experimental biped was able to learn the closed-chain kinematics necessary to shift body weight side-to-side while maintaining good foot contact. Also, it was able to learn the quasi-static balance required to avoid falling forward or backward while shifting body weight side-to-side at different speeds. There were, however, many limitations (limited step length, slow walking, no adaptation for left-right balance, no possibility of walking on sloping surfaces). Hence upgrading and improvement of this approach were proposed in (Kun et al. (1999)). The new dynamically balanced scheme for handling variable-speed gait was proposed based on the preplanned but adaptive motion sequences in combination with closed-loop reactive control. This allows the algorithm to improve the walking performance over consecutive steps using adaptation, and to react to small errors and disturbances using reactive control. New sensors (piezoresistive accelerometers and two solid-state rate gyroscopes) are mounted on the new UNH biped (Figure 1). The complete control structure consists of high-level and low-level controllers (Figure 2) The control structure on high-level control includes 7 components: gait generator, simple kinematics block and 5 CMAC controllers. The operation of the gait generator is based on simple heuristics and an appropriate biped model. The CMAC neural networks are used for compensation

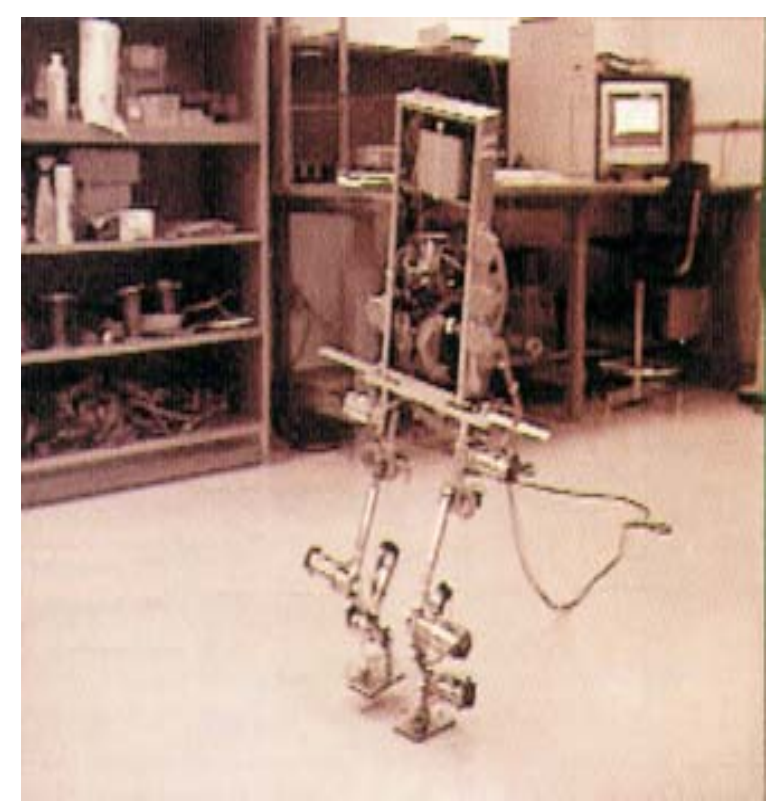

Fig. 1. The UNH biped walking

of right and left lift-lean angle correction, reactive front-back offset, right-left lean correction, right and left ankle - $Y$ correction and front-back lean correction. Training of neural networks is realized through the process of temporal difference learning using information about ZMP from robot foot sensors. The five CMAC neural networks were first trained during repetitive foot-lift motion similar to marching in place. The control structure on the lower control level includes reactive lean angle control, together with a PID controller. The experimental results indicate that the UNH biped robot can walk with forward velocities in the range of $21-72 \mathrm{~cm} / \mathrm{min}$, with sideways leaning speed in the range of $3.6-12.5 \circ / \mathrm{s}$. The proposed controller could be used as a basis for similar controllers of more complex humanoid robots in the future research. However, this controller is not of a general nature, because it is suitable only for the proposed structure of biped robot and must be adapted for the bipeds with different structures.

In paper(Hu et al. (1999)), self-organizing CMAC neural network structure was proposed for biped control based on a data clustering technique together with adaptation of the basic control algorithm. In this case, memory requirements are drastically reduced and globally asymptotic stability is achieved in a Lyapunov sense. The structural adaptation of the network centres is realized to ensure adaptation to unexpected dynamics. Unsupervised learning using CMAC can be implemented with a Lyapunov trajectory index. The distance between the input vector and the centre vectors of the CMAC is calculated, then the memory cells corresponding to the centres (hit by the input) are found, and finally, computation of the CMAC output by a linear combination of CMAC basis functions and weights of the memory 
HIGH-LEVEL CONTROL

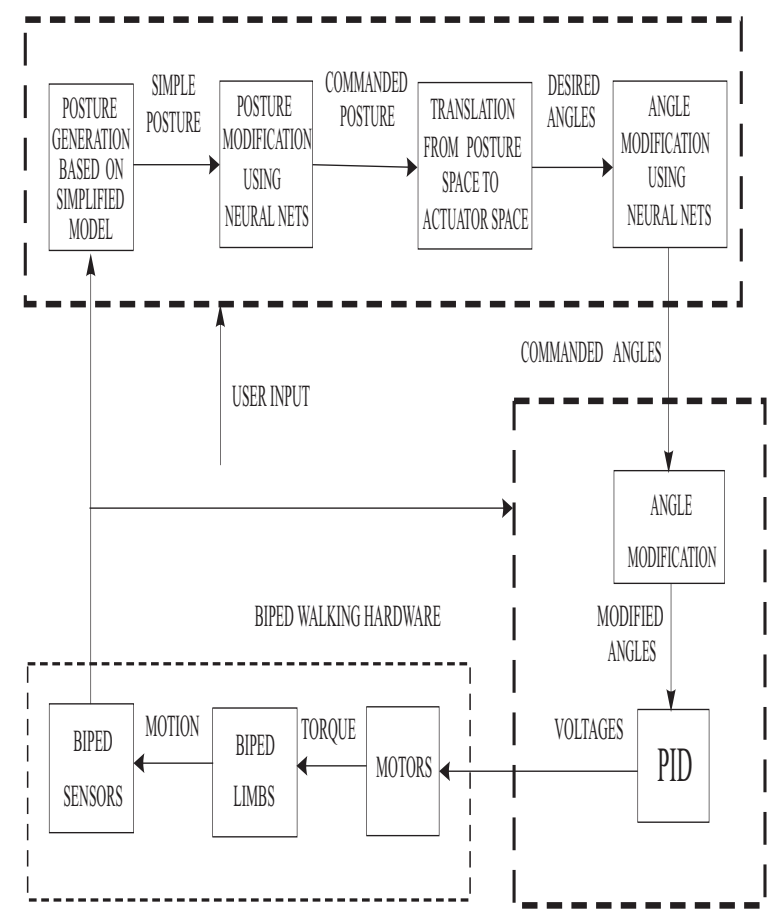

LOW.LEVEL CONTROL

Fig. 2. Block Diagram of Overall Biped Control System

cell is achieved. The weights in the fired memory cells are updated by unsupervised learning. The approach is verified through simulation experiments on a biped with 7 DOFs. An important characteristic of this approach is the inclusion of adaptation for CMAC and PID controllers with a moderate increase of controller complexity to handle disturbances and environmental changes.

Wang et al.1992 have developed a hierarchical controller for a three-link two-legged robot. The approach uses the equations of motion, but only for the training of the neural networks, rather than to directly control the robot. The authors used a very simplified model of biped with decoupled frontal and sagittal planes. There are 3 neural networks (multilayer perceptrons) for control of leg on the ground, control of leg in the air, and for body regulation. Training algorithm is a standard back- propagation algorithm based on the difference between the decoupled supervising control law and output of all three neural networks. There are no feedback in real-time control, and this is a great problem in the case when the system uncertainties exist.

Doerschuk et al. 1998 presented an adaptive controller to control the movement of simulated jointed leg during a running stride (uniped control). The main idea of this approach is the application of modularity, i.e. the use of separate controllers for each phase of the running stride (take-off, ballistic, landing), thus allowing each to be optimized for the specific objective of its phase. In the take-off phase, the controller's objective is to realize inverse feedforward control (for desired height, distance and angular momentum it is necessary to produce control signals that achieve these objectives). Three different types of neural networks are investigated (multilayer perceptrons, CMAC, and neuro-fuzzy nets). It was concluded that neuro-fuzzy nets achieve more accurate results than the other two methods. The neuro-fuzzy take-off controller controls very accurately the value of angular momentum of the stride after only two learning iterations. The ballistic controller controls the movement of the leg while the foot is in the air. In this case, ballistic controller combines neural network learning with the conventional PD control. The controller learns the dynamic model of leg from experience generated by the PD controller and improved upon its performance. The CMAC controller is used for neural network learning part with the possibility to very accurately control the movement of the leg along a target trajectory even during the first attempt. Ballistic learning is accomplished on-line without the need for precomputed examples. This enables effective adaptability of humanoid robot to various changes and new conditions.

The neural networks can be effectively used to generate trajectories (gait) of humanoid robots (Kurematsu et al. (1991);Juang and Lin (1996)). Kurematsu 1991 proposed a multi-layered network by using the centre of gravity concept in trajectory generation. For example, Juang and Lin 1996 used the back propagation through time algorithm for gait synthesis of a biped robot. Due to a high number of DOFs of the biped, it is difficult to get a high nonlinear model of the biped. Hence, the complex inverse dynamic computations were eliminated by using linearised inverse biped model. The neural controller is a three-layer feedforward network. The simulation results show that the neural network as open-loop controller can generate control sequences to drive the biped along a prespecified trajectory. This algorithm can also be used for the slope surface training.

Fujitsu Laboratories, Ltd. has developed the world's first learning system for humanoid robots that uses a dynamically reconfigurable neural network (www.fujitsu.com) to enable the efficient learning of movement and motor coordination. This achievement is a significant leap forward in the development of humanoid robots, making the generation of motion in a humanoid robot, for which complex controls are required, a dramatically faster and simpler process. Fujitsu's new technology is based on Central Pattern Generator (CPG) networks, which mathematically simulate the neural oscillator found in vertebrates. 
This is combined with a Numerical Perturbation Method (NP) that quantifies the configuration and connection-weight status of the network. This combination, known as CPG/NP learning, is optimized in the new technology. In addition, Fujitsu simultaneously developed a software program, known as the Humanoid Movement-Generation System, which enables humanoid robots to learn a wide range of movements. Key Features of the New System are: 1) Unprecedented learning flexibility The system enables unprecedented flexibility in learning movements, thanks to a neural network that is dynamically reconfigured using multiple central pattern generators and the numerical perturbation method, which selects the best movements for the humanoid robot. The central pattern generators generate motion in the robot using self-induced oscillations. These are evaluated using a pre-set evaluation function that determines whether the movement is correct or incorrect.The robot's movements are altered by slightly changing the connection weights of the central pattern generators, and this process is repeated until the robot is moving well. At that point, with Fujitsu's newly developed method, the numerical perturbation method will, as needed, either generate a new central pattern generator or reconfigure existing combinations, and thereby automatically select the most appropriate movement as the neural network is being dynamically reconfigured.The learning process is not simply a matter of changing the connection weights; the structure of the network itself changes so that it can learn a variety of complex motions.2)Fast learning and execution with minimal software.This approach to development greatly minimizes the size of software code involved in motion control, cutting it to less than one-tenth that used in conventional systems. Learning time is reduced to an astonishing $10^{-30 t h}$ of the time previously required (assuming a robot with 20 movable joints). This enables the robot to learn to adapt and instantly react as it moves about in a virtual real-world environment. 3)Humanoid motion-generation system The new technology also forms the basis for a prototype humanoid motion-generation system. Comprised of a neural network display/edit unit, a robot simulation unit, and a mechanical interface (Figure 4 ), this system enables even people without any expertise in the field of dynamics to generate the desired movements in humanoid robots.

Arsenio 2004, also used neural oscillators, becauser they offer a natural tool for exploiting and adapting to the dynamics of the controlled system. The Matsuoka neural oscillator consists of two neurons inhibiting each other mutually. The capability of entraining the frequency of the input signal or resonance modes of dynamical systems have been increasingly used in robotics' mechanisms, to ac- complish complex tasks. However, the application of Matsuoka neural oscillators as controllers requires the knowledge of the range of values for the parameters for which the system oscillates, and the warranty of stability. Thus, In this paper, stability and tuning of Matsuoka neural oscillators are shown, and a careful analysis of its behavior on the time-domain is presented. The proposed method is applied on a Humanoid Robot for playing musical instruments.

\section{FUZZY CONTROL ALGORITHMS IN HUMANOID ROBOTICS}

Some researchers used the fuzzy logic (Zhou and Meng (2000); Yang and Low (2002);Ivanescu et al. (2001)) as the methodology for biped gait synthesis and control of biped walking. Fuzzy logic was used mainly as part of control systems on the executive control level, for generating and tuning PID gains, fuzzy control supervising, direct fuzzy control by supervised and reinforcement error signals.

The problem of biped gait synthesis using the reinforcement learning with fuzzy evaluative feedback is considered in paper (Zhou and Meng (2000)). As first, initial gait from fuzzy rules is generated using human intuitive balancing scheme. Simulation studies showed that the fuzzy gait synthesizer can only roughly track the desired trajectory. A disadvantage of the proposed method is the lack of practical training data. In this case there are no numerical feedback teaching signal, only evaluative feedback signal exists (failure or success), exactly when the biped robot falls (or almost falls) down. Hence, it is a typical reinforcement learning problem. The dynamic balance knowledge is accumulated through reinforcement learning constantly improving the gait during walking. Exactly, it is fuzzy reinforcement learning that uses fuzzy critical signal. For human biped walk, it is typical to use linguistic critical signals such as "near-fall-down", "almost-success", "slower", "faster", etc. In this case, the gait synthesizer with reinforcement learning is based on a modified GARIC (Generalized Approximate Reasoning for Intelligent Control) method. This architecture of gait synthesizer consists of three components: action selection network (ASN), action evaluation network (AEN), and stochastic action modifier (SAM) (Figure 3). The ASM maps a state vector into a recommended action using fuzzy inference. The training of ASN is achieved as with standard neural networks using error signal of external reinforcement. The AEN maps a state vector and a failure signal into a scalar score which indicates the state goodness. It is also used to produce internal reinforcement. The SAM uses both recommended action and internal reinforcement to 
produce a desired gait for the biped. The reinforcement signal is generated based on the difference between desired ZMP and real ZMP in the $\mathrm{x}-$ y plane. In all cases, this control structure includes on-line adaptation of gait synthesizer and local PID regulators. The approach is verified using simulation experiments. In the simulation studies, only even terrain for biped walking is considered, hence the approach should be verified for irregular and sloped terrain. where $X z m p, Y z m p$ are the

Failure signal Rxl

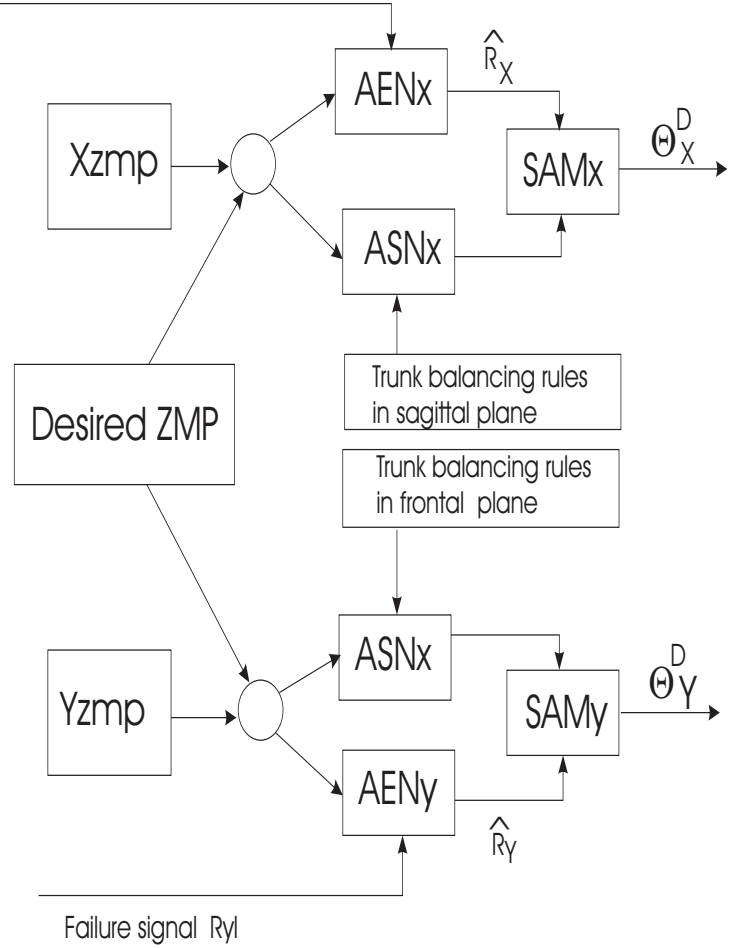

Fig. 3. The architecture of the reinforcement learning based gait synthesizer

ZMP coordinates; $\theta_{z m p}^{d}, \theta_{z m p}^{d}$ are the desired joint angles of the biped gait.

In paper (Yang and Low (2002)), conventional fuzzy controller for position/force control of robot leg is proposed and experimentally verified. This intelligent walking strategy is specially intended for walking on rough terrain.

A main problem in the synthesis of fuzzy control algorithms for biped robots remains the inclusion of dynamic model and learning capabilities in order to obtain exact tracking of biped trajectories as well as the steps with greater speed, preserving dynamic stability of the biped gait.

Sabourin et al. 2004 proposed an intuitive and fuzzy control method for regulation dynamic walking on under-actuated bipedal robot without reference trajectories. The intuitive control method is based on alternate periods of active and passive stages. The active periods are composed by a succession of impulsive torques and PD control which allow to ensure the stability and the propulsion of the robot. This control strategy makes it possible to produce the dynamic walking of the under-actuated robot qith as many steps as we desire. The extension of this method includes computation of amplitude of impulsive torques by using a Fuzzy Inference System (FIS),which permits to formalize the human intuitive knowledge.

\section{GENETIC APPROACH IN HUMANOID ROBOTICS}

It is considered that GA can be efficiently applied for trajectory generation of the biped natural motion on the basis of energy optimization (Arakawa and Fukuda (1997); Capi et al. (2001)), as well as for walking control of biped robots (Cheng and Lin (1997)) and for generation of behaviour-based control of these systems (Pettersson et al. (2001)).

The hierarchical trajectory generation (Arakawa and Fukuda (1997)) method consists of two layers, one is the GA level which minimises the total energy of all actuators and the other is the evolutionary programming (EP) layer which optimises the interpolated configuration of biped locomotion robots. The trajectory of biped is generated using ZMP stability conditions. The chromosome on the EP level represents the interpolated configuration expressed by 12 state variables (angles) of the biped. Also, a chromosome in a GA level consists of two parts, the first of them representing the set of interpolated configurations, while the second part includes a bit which represents the effectiveness of the configuration ( 0 or 1$)$. The process runs in a cyclic procedure through the application of mutation and selection at the EP level, transfer of generated interpolated configuration into the GA level, and complete evolution process through crossover, mutation, evaluation and selection at the GA level. The fitness function at the GA level is connected to the optimisation of total robot energy in order to ensure the natural movement of the biped. The fitness function also contains some constraints related to the robot motion. The final result represents an optimised trajectory similar to natural human walking, which was demonstrated by the simulation experiment.

A typical example of the application of GA in humanoid robotics was presented in paper (Capi et al. (2001)), where the main intention was the optimal gait synthesis for biped robots. The proposed method can easily be applied onto other tasks like overcoming obstacles, going down stairs, etc. In solving these optimization tasks, the most important constraint included is the stability, which is verified through the ZMP concept. To ensure a stable motion, the jumping of the ZMP is realized by accelerating the body link. GA makes easy handling of the constraints by using the penalty 
function vector, which transforms a constrained problem into an unconstrained one. The optimisation process is based on considering two different cost functions: minimisation of consumed energy (CE) and minimisation of torque change (TC). In this optimisation process, some constraints are included such as the stability conditions defined by ZMP to be within the sole length. The block diagram of the GA optimisation method is presented in Figure 4.

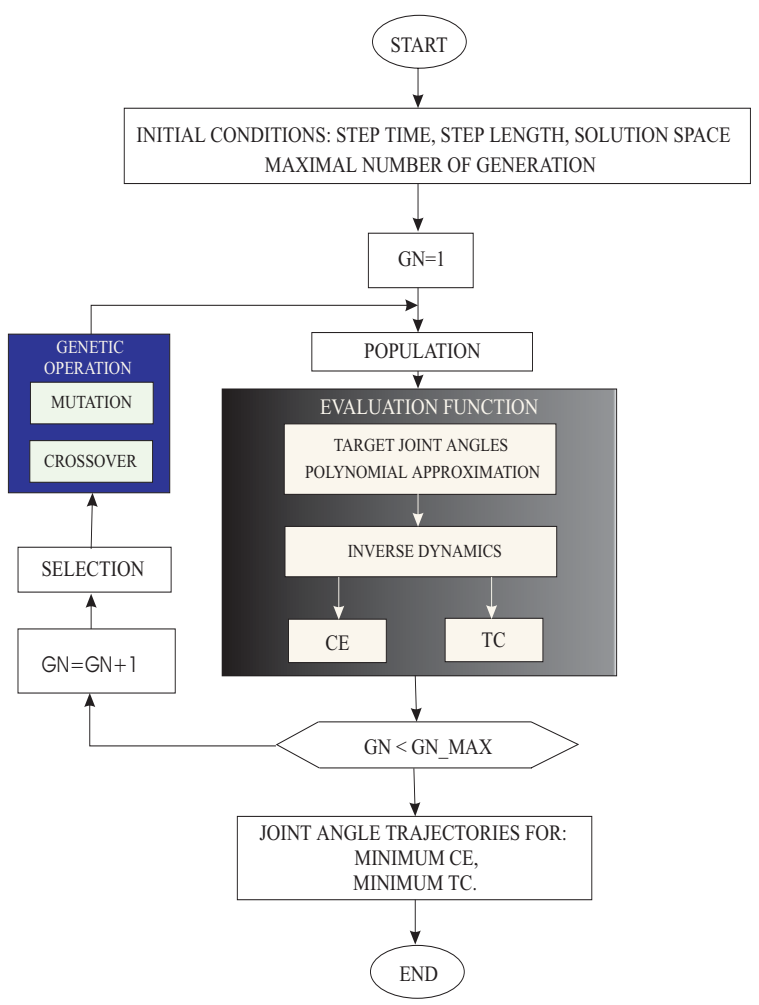

Fig. 4. Block Diagram of the GA optimization process

Based on the initial conditions, the initial population, represented by the angle trajectory in the form of a polynomial of time, is created. Its range is determined on the basis of the number of angle trajectory constraints and the coefficients are calculated to satisfy these constraints. In the simulation experiments, the parameters of real humanoid robot "Bonten-Maru I" are used. For the optimisation of the cost function, a real-value GA was employed in conjunction with the selection, mutation and crossover operators. GA converges within 40 generations, while the maximum number of generations is used as the termination function. Based on simulation, the biped robot posture is straighter, like the human walking when the $\mathrm{CE}$ is used as cost function. The torques change more smoothly when minimum $\mathrm{TC}$ is used as a cost function.

However, for the real-time applications, some process of GA optimisation is time-consuming (in this case, optimisation process needs 10 min- utes). Hence, the author considered teaching a RBFNN (Radial Basis Function Neural Networks) based on GA data. When the biped robot was to walk with a determined velocity and step length, the RBFNN input variable would be step length and step time, while the output variables of the RBFNN were the same as the variables generated by GA. Simulations showed good results generated by RBFNN in a very short time (only 50 $\mathrm{ms})$.

Another example is the application of GA to PD local gain tuning and determination of nominal trajectory for dynamic biped walking (Cheng and Lin (1997)). The biped with 5 links is considered. In the proposed GA, 19 controller gains and 24 final points for determination of nominal trajectory are taken into account. In order the biped body be in the vertical plane during walking, some constraints related to the fixation of joint angles are realised. Hence, it is possible to reduce the number of parameters of nominal trajectory for optimisation by 6 parameters. Designs to attain different goals, such as the capability of walking on an inclined surface, walking at high speed, or walking with specified step size, have been evolved with the use of GA. The research showed excellent simulation results in the evaluation of control parameters, as well as in optimisation of the mechanical design of biped.

The main problem of GA application in humanoid robotics represents the coping with the reduction of GA optimisation process in real time.

\section{HYBRID INTELLIGENT APPROACHES IN HUMANOID ROBOTICS}

Because their complementary capabilities hybrid intelligent methods have also found their place in the research of gait synthesis and control of humanoid robots.

In paper (Juang (2000)), a learning scheme based on a neuro-fuzzy controller to generate walking gaits, is presented. The learning scheme uses a neuro-fuzzy controller combined with a linearised inverse biped model. The training algorithm is back propagation through time. The linearised inverse biped model provides the error signals for back propagation through the controller at control time instants. For the given prespecified constraints such as the step length, crossing clearance, and walking speed, the control scheme can generate the gait that satisfies all the mentioned constraints. Simulation results are verified for a simple structure of five-link biped robot.

In paper (Ferreira et al. (2004)), an adaptive neural-fuzzy walking control of an autonomous biped robot is proposed. This control system uses 
a feed forward neural network based on nonlinear regression. The general regression neural network is used to construct the base of an adaptive neurofuzzy system. The neural network uses an iterative grid partition method for the initial structure identification of the controller parameters. In this perspective, the proposed control system combines the fuzzy expert knowledge and neural network into an adaptive neuro-fuzzy inference system.

To control the biped in its different motions, it is proposed one adaptive neuro-fuzzy inference controller with four inputs and seven outputs. The output values are the angles of the joints of the walking robot. In the proposed method, the neuro-fuzzy system learns with the training data set derived from the expert knowledge of the biped motion control. After the initial parameter values of the network are defined they are readjusted to reduce the error values of the joint angles positions. This process is repeated until some minimum value of the error function or a predefined epoch number have been reached. The adjustment of the neuro-fuzzy parameters was performed by a hybrid technique that uses the backpropagation and the mean- squared error. The parameters of the antecedent part are tuned using the gradient descent technique. The parameters of the consequent part are learned by the least square estimate through the sequential formulas making the algorithm more efficient. After the adaptive neuro-fuzzy controller is trained, the controller can be used to control the biped motion. Comparison results are done between the proposed method and the ANFIS tool provided in the fuzzy MATLAB toolbox. The robot's control system uses an inverted pendulum to balance of the gaits. These results show the effectiveness of the proposed initial structure identification. Also the proposed membership functions for the antecedent part, gave more flexibility to the learning stage contributing to reduce the learning error. The robot can walk in horizontal and sloping planes.

The GA has been efficiently applied in robotic neural approaches, as in the case of the neuroGA controller for visually-guided swing motion of a biped with 16 DOFs (Nagasaka et al. (1997)). The aim of this robot task is learning of swing motion by neural network using visual information from a virtual working environment. As is known, GA requires a lot of computing time in order to evaluate the fitness function for each individual in a population. Hence, it is not desirable to use direct execution of the working task on a real biped because of task complexity and inaccuracies of the sensors. Instead of a real biped, virtual working environment is used for acceleration of the learning process. As the learning process is transferred from the virtual environment to the real robot, the difference existing between these two systems is neutralized by generalization capabilities of the neural network. The aim of learning for visually guided swing motion is increasing the swing amplitude by skillful change of the gravity center of the biped robot in the direction of swing radius, caused by dynamic change of the environment recognized by the vision sensor. The input to the network represents sensor information from the vision sensor, while the output of the neural network is the knee angles of the biped (Figure 4). GA optimizes the three sets of weighting factors of this 4-layer neural network. At the output of the network, the data are transformed into joint angles and then using limiters of angular velocities (to avoid extreme changes of joint angles), the knee joint angle is calculated. The genotype is represented by a sequence of weighting factors. The number of individuals in the initial population is 200. The fitness function is represented by the height of the center of gravity in the initial and final pose. The evolution simulation experiment is terminated when the number of alternations in generations reaches 50 . The results show the efficient learning of swing motion through successive generation that is verified through generalization experiments on the real robot biped.

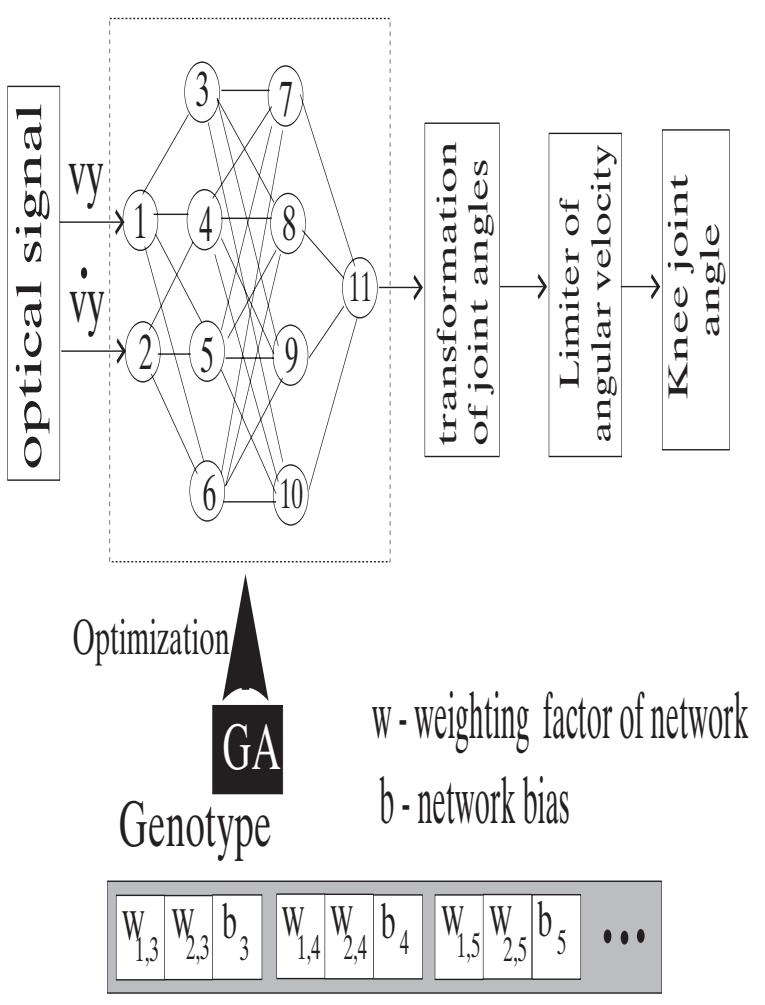

Fig. 5. Neuro - GA approach for optimization of robot swing motion

In paper (Fukuda et al. (1997)) the authors deal with a GA application for the determination of weighting factors of a recurrent neural network in order to generate a stable biped gait. When the 
biped robot walks on the ground which has some gradients, the optimal trajectory is not known, hence the optimal trajectory of ZMP is not realized. Because of that, the reinforcement learning is used by applying a recurrent neural network. Recurrent neural network is chosen in order to select best biped configuration (desired joint position and velocity) using ZMP as stabilization index. This type of neural network was chosen because the output of the network generates the dynamic output data for static inputs and can describe time records easily. The input to the network is the information about position of ZMP taken from the force sensor, while the output of the network is the correction angles and correction velocities needed for a stable motion. The ZMP is calculated using the values from force sensors at each sole and values of joint angles. Only self-mutation is used from the set of genetic operators based on addition of the Gauss noise with multiplication by the value of fitness function. The elite selection is chosen, while the fitness function is defined by the sum of squares of the deviations of the desired coordinates from the ZMP coordinates. In both single-support and double-support phases of walking the algorithm calculates the ZMP by using values from four force sensors at each sole, while correction to actuation angles and velocities is determined by recurrent neural networks with the ZMP being within the supporting area of the sole of the robot. The block diagram of the stabilisation biped control is shown in Figure 5 , where $\theta$ ,$\dot{\theta}$ are the joint angles and velocities; $\theta_{D}, \dot{\theta}_{D}$ are the desired joint angles and velocities; $U$ is the control signal; $F$ is the foot force. The motion on inclined surfaces is investigated with initial population of 50 different individuals. It has been shown that the use of this approach yields a stable biped gait.

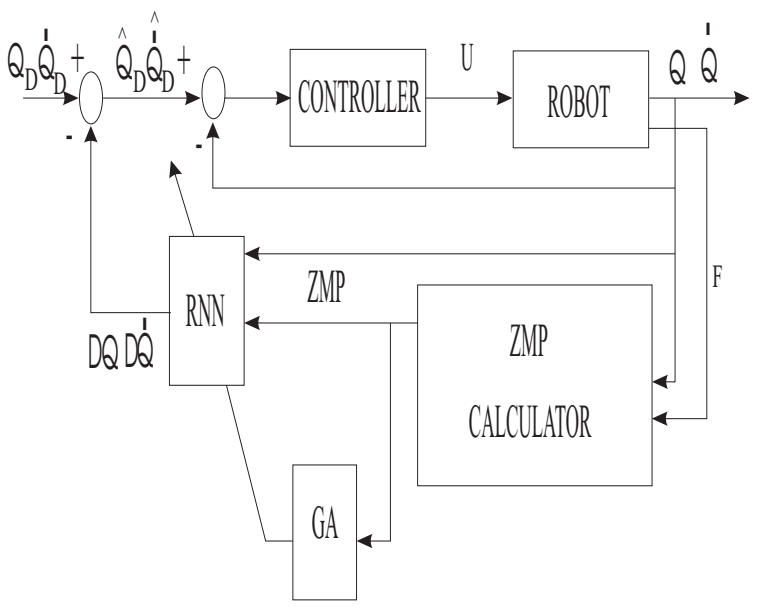

Fig. 6. Block Diagram of Stabilization Biped Control

Reil and Husbands 2002 proposed an evolutionary approach for the biped controller based on dynamic recurrent neural network. Each neural network consists of 10 fully interconnected neurons. The first 6 neurons represent motor neurons because that control biped actuators (the biped has 6 DOFs). Their outputs are scaled to map to the angle limits. The GA has a task to optimize the weighting factors, time constant, and bias of activation functions for the chosen neurons. Parameter values are coded using real numbers with different ranges for each type. Each population consists of 50 individuals. The Rank-Based selection is used for generating new generations with a fittest fraction. From the genetic operators, only mutation with small rate is applied. The fitness function considers two components: 1) the minimisation of travelling distance from the origin; 2) the gravity centre can not be below a certain height. The fraction of evolutionary runs leading to stable walkers was $10 \%$, of which the average walking distance was $20.577 \mathrm{~m}$. All controllers in the simulation experiments walked in a straight line without the use of proprioceptive inputs and without active balance control. because of the application of the mentioned fitness function. The authors proposed an additional criterion for fitness function in order to reward cycling activity. In this way, the proportion of successful runs is increased to $80 \%$ but without improvement of the overall time efficiency. In order to achieve walking on a rough terrain, some set of simulation experiments with inclusion of sensor signals as input to neurons of the neural network, are realized. These preliminary experiments on the integration indicate that cyclic walking activity can indeed be modified by external stimuli in a meaningful way.

A very interesting approach was proposed in paper (Endo et al. (2002)), using the ideas of artificial life. The main idea is to optimize both the morphology and control of biped walking at the same time, instead of optimizing the walking behaviour for the given hardware. It was shown that the generated robots have diverse morphologies and control systems, while their walking is fast and efficient. Both the morphology and neural systems are represented as simple large tree structures that are optimized simultaneously. From the morphology side, the lengths of the lower and upper limbs are optimized. Two types of control systems are analyzed: the one based on neural network and the other based on neural oscillator. The input to the neural network represents the velocity, acceleration and ZMP position, while the output of the network represents the joint angles. It is a layered neural network with a pair of hidden layers. The chromosome includes the following parameters for optimization: information on initial angle and velocity, length of each link and weights of each neuron in the neural network. The simulation experiments with population size 
of 200 individuals and 600 generations were realized using standard genetic operators. In the first phase of GA, the fitness function was the distance between the center of mass of the robot and the initial point. In the second phase, two fitness functions were evaluated based on the efficiency and stability of walking. The preferred solution has appropriate locomotion and morphology. As the other solution for control algorithm, neural oscillator was used, because the biped walking is a periodical and symmetrical solution. Neural oscillator generates the rhythm for the biped walking. In this case, it is not necessary to use a largesize GA chromosome, as was the case with neural networks. The structure of the neural oscillator represents some kind of recurrent neural network dynamic state, while the other parameters of GA optimization process are the same as in the previous case. Therefore, a larger dynamic model of biped can be applied to the model with a neural oscillator. It has been shown that there is a close relation between the morphology and locomotion.

\section{CONCLUSIONS}

In spite of the intensive development and experimental verification of various humanoid robots, it is important to further improve their capabilities using advanced hardware and control software solutions to make humanoid robots more autonomous, intelligent and adaptable to the environment and humans. The presented survey indicates that the intelligent techniques, if applied in an appropriate manner, can be very powerful tools for attaining these goals. The neural networks were used for the synthesis and on-line adaptation of biped gait, as well as for the control of humanoid robots to ensure static and dynamic balance during the process of walking and running on the terrain with different environment characteristics. Besides, the inclusion of complex nonlinear models in real-time control, limited realized steps and slow walking are the problems in implementation of connectionist control algorithms. Fuzzy logic was used mainly as part of control systems on the executive control level, for generation and efficient tuning of PID gains and direct fuzzy control by supervised and reinforcement error signals. The GA represents an efficient tool for searching the optimized solutions of gait synthesis and biped control, the main problem being how to cope with the reduction of GA optimization process in real time and preserve stability of the motion. The hybrid methods using complementary characteristics of intelligent techniques have a great potential in the field of intelligent humanoid robots.

\section{REFERENCES}

Arakawa, T. and T.Fukuda (1997), Natural Motion Generation of Biped Locomotion Robot Using Hierarchical Trajectory Generation Method Consisting of GA,EP Layers, In:Proceedings of the IEEE International Conference on Robotics and Automation, Albuquerque, pp.211-216.

Arsenio A.M. (2004). On stability and Tuning of Neural Oscilators: Application to Rhythmic Control of a Humanoid Robot". In:Proceedings of the 2004 IEEE International Joint Conference on Neural Networks, Budapest, Hungary.

Capi, G., Y.Nasu, L.Barolli, M. Yamano, K.Mitobe and K. Takeda (2001), A Neural Network Implementation of Biped Robot Optimal Gait During Walking Generated by Genetic Algorithm, In:Proceedings of the 9th Mediterranean Conference on Control and Automation, Dubrovnik, Croatia.

Cheng, M-Y. and C-S.Lin (1997). Genetic Algorithm for Control Design of Biped Locomotion, Journal of Robotic Systems, 14(5). pp.365-373,

Doerschuk, P.I., W.E.Simon, V.Nguyen and A.Li (1998). A Modular Approach to Intelligent Control of a Simulated Jointed Leg, IEEE Robotics and Automation Magazine, 5(2), pp.12-21.

Endo, K., F.Yamasaki,T.Maeno and H.Kitano (2002). A Method for Co-Evolving Morphology and Walking Pattern of Biped Humanoid Robot, In: Proceedings of the 2002 IEEE International Conference on Robotics and Automation, Washington, D.C., USA, pp. 2159-2164.

Ferreira, J.P., T.G..Amaral, V.F.Pires, M.M.Crisostomo and A.P.Coimbra (2004).A Neural-fuzzy Walking Control of Autonomous Biped Robot, In:Proceedings of the 10th International Symposium on Robotics with Applications, Seville, Spain.

Fukuda, T., Y.Komata and T.Arakawa (1997). Stabilization Control of Biped Locomotion Robot Based Learning with GAs having Selfadaptive Mutation and Recurrent Neural Network. In:Proceedings of the IEEE International Conference on Robotics and Automation, Albuquerque, USA, pp.217-222.

Goswami A. (1999). Postural Stability of Biped Robots and the Foot-Rotation Indicator Point, International Journal of Robotics Research, 18(6),pp.523-533.

Hu, J., J.Pratt and G.Pratt (1999). Stable Adaptive Control of a Bipedal Walking Robot with CMAC Neural Networks. In:Proceedings of the 1999 IEEE International Conference on Robotics and Automation, Detroit, USA. pp.1950-1956.

Huang, Q., Y.Nakamura and T.Inamura (2001). Humanoids Walk with Feedforward Dynamic Pattern and Feednack Sensory Reflection. 
In:Proceedings of the 2001 IEEE International Conference on Robotics and Automation, Seoul,Korea, pp.4220-4225.

Ivanescu, M., A.M.Popescu and D.Popescu (2001). Moving Target Interception for a Walking Robot by Fuzzy Observer and Fuzzy Controller. Proceedings of the 4th International Conference on Climbing and Walking Robots, Karlsruhe, Germany.

Juang, J-G. and C-S.Lin (1996). Gait Synthesis of a Biped Robot Using Backpropagation Through Time Algorithm, In:Proceedings of the IEEE International Joint Conference on Neural Networks, Washington, D.C., USA, pp.1710-1715.

Juang J-G(2000). Fuzzy Neural Network Approaches for Robotic Gait Synthesis.IEEE Transactions on Systems, Man and Cybernetics, Part B: Cybernetics, 30(4), pp. 594-601.

Kitamura, S., Y.Kurematsu and Y.Nakai (1988). Application of the neural network for the trajectory planning of a biped locomotion robot, Neural Networks, 1,pp.344-356.

Kun , A.L. and W.T.Miller,III (1999).. Control of Variable - Speed Gaits for a Biped Robot.IEEE Robotics and Automation Magazine, 6(3),pp. 19-29.

Kurematsu, Y., O.Katayama, M.Iwata and S.Kitamura (1991). Autonomous Trajectory Generation of a Biped Locomotive Robot. In:Proceedings of the IEEE International Joint Conference on Neural Networks, pp.1983-1988.

Marchese, S., G.Muscato and G.S.Virk (2001). Dynamically Stable Trajectory Synthesis for a Biped Robot During the Single-Support Phase, In" Proceedings of the 2001 IEEE/ASME International Conference on Advanced Intelligent Mechatronics, Como, Italy, pp.953-958.

Miller , III, W.T. (1994). Real-Time Neural Network Control of a Biped Walking Robot, IEEE Control Systems Magazine, pp.41-48.

Miller, III, W.T., F.H.Glanz and L.G.Kraft (1987). Application of a General Learning Algorithm to the Control of Robotic Manipulators. International Journal of Robotics Reserach, 6(2).

Nagasaka, K., A.Konno, M.Inaba and H.Inoue (1997). Acquisition of Visually Guided Swing Motion Based on Genetic Algorithms and Neural Networks in Two-Armed Bipedal Robot. In:Proceedings of the IEEE International Conference on Robotics and Automation, Albuquerque, USA, pp.2944-2949.

Pettersson, J., H.Sandholt and H.Wahde (2001). A Flexible Evolutionary Method for the Generation and Implementation of Behaviours for Humanoid Rpbots. In:Proceedings of the IEEE$R A S$ International Conference on Humanoid Robots HUMANOIDS 2001, Tokyo, Japan.

Raibert M. (1986) . Legged Robots That Balance, The MIT Press, Cambridge, USA.
Reil, T. and P.Husbands (2002). Evolution of Central Pattern Generators for Bipedal Walking in a Real-Time Physics Environment. IEEE Transactions on Evolutionary Computation, 6(2), pp.159-168.

Sabourin, C., O. Bruneau and J.-G. Fontaine (2004). Intuitive and Fuzzy Control of an Under-actuated Bipedal Robot Without References Trajectories. In: Proceedings of the 11th World Congress in Mechanism and Machine Science, IFToMM 2004, Tianjin, China, China Machinery Press.

Salatian, W. and Y.F.Zheng (1992). Gait synthesis for a biped robot climbing slopimg surfaces using neural networks - I. Static Learning. In:Proceedings of the IEEE International Conference on Robotics and Automation, Nice, France,pp.2601-2606.

Salatian, W. and Y.F.Zheng (1992). Gait synthesis for a biped robot climbing slopimg surfaces using neural networks - II. Dynamic Learning. In:Proceedings of the IEEE International Conference on Robotics and Automation, Nice, France,pp.2607-2611.

Salatan, W., K.Y.Yi and Y.F.Zheng (1997). Reinforcement Learning for a Biped Robot to Climb Sloping Surfaces. Journal of Robotic Systems, 14(4),pp.283-296.

Vukobratovic, M., B.Borovac and D.Surdilovic (2002). Zero-Moment Point - Proper Interpretation and the Application in Gait Control. Intelligent Journal Engineering 86 Automation Problems, 3,pp.3-14.

Wang, H., T.T.Lee and W.A.Gruver (1992). A Neuromorphic Controller for a Three Link Biped Robot. IEEE Transactions on Systems, Man and Cybernetics, 22(1),pp.164-169.

www.androidworld.com

www.fujitsu.com

Yang, A. and K.H.Low (2002). Fuzzy Position/Force Control of a Robot Leg with a Flexible Gear System. In:Proceedings of the 2002 IEEE Conference on Robotics and Automation, Washington, D.C., USA,pp.2159-2164.

Zheng, Y.P. and J.Shen (1990). Gait Synthesis for the SD-2 Biped Robots to Climb Sloping Surface.IEEE Transactions on Robotics and Automation, 6(1), pp.86-96.

Zhou C. and Q.Meng (2000). Reinforcement Learning with Fuzzy Evaluative Feedback for a Biped Robot. In:Proceedings of the IEEE International Conference on Robotics and Automation, San Francisko, USA, pp.3829-3834.

Zhou, D. and K.H.Low (2001). Combined Use of Ground Learning Model and Active Compliance to the Motion Control of Walking Robotic Legs. In:Proceedings of the 2001 IEEE International Conference on Robotics and Automation, Seoul, Korea, pp. 3159-3164. 